\title{
Effect of Alkali Metal Hydroxides on the Morphological Development and Optical Properties of Ceria Nanocubes Under Hydrothermal Conditions
}

\author{
Özlem Kepenekçi, Mehtap Emirdag-Eanes*, and Mustafa M. Demir* \\ Izmir Institute of Technology, Department of Chemistry, Gülbahçe köyü, Urla, 35430 Izmir, Turkey
}

\begin{abstract}
Nanocrystalline cerium(IV) oxide $\left(\mathrm{CeO}_{2}\right.$, ceria) particles were produced via the hydrothermal treatment of cerium nitrate hexahydrate with various alkali metal hydroxides ( $\mathrm{MOH}: \mathrm{M}=\mathrm{Li}, \mathrm{Na}, \mathrm{K})$. Experimental conditions such as $[\mathrm{MOH}]$, reaction temperature, and reaction time were studied. Particle morphology as well as size of crystallites was precisely controlled by choice of experimental conditions. While rod-shaped particles were obtained at $120{ }^{\circ} \mathrm{C}$, well-defined nanocubes were formed at higher temperatures regardless of the choice of $\mathrm{MOH}$. Examination of particle growth kinetics, in the final stages of crystallization, showed that particle growth rate is controlled by two different mechanisms. Grain boundary diffusion controls the particle growth in the presence of $\mathrm{NaOH}$ with an activation energy of $113.8 \mathrm{kj} / \mathrm{mol}$ and surface diffusion for $\mathrm{LiOH}$ ad $\mathrm{KOH}$ with the activation energy of 43.0-150.9 kj/mol, respectively. In addition, the particles exhibit strong violet and blue emissions at $400 \mathrm{~nm}$ and $370 \mathrm{~nm}$. The former emission originates from excitation of a wide band gap of $\mathrm{CeO}_{2}$. The latter one is attributed to the trivalency of the cerium ion and appears to be sensitive to all the experimental conditions studied. Both extending reaction time and increasing temperature reduce the intensity of the $370 \mathrm{~nm}$ emission and increase the intensity of the $400 \mathrm{~nm}$ emission.
\end{abstract}

Keywords: Ceria, Crystal Growth, Growth Kinetics, Nanoparticles, Semiconductor.

\section{INTRODUCTION}

Nanoscale semiconductor particles are of great interest due to their unique morphology dependent (size and shape) physical properties. ${ }^{1-3}$ Therefore, synthetic methods have been developed to tailor the morphology of these particles making them useful in practical applications. In particular, there is an increasing interest in preparation of cerium oxide (ceria) that has recently been used in various fields such as an oxygen ion conductor in solid-oxide fuel cells ${ }^{4}$ and as an oxygen pump due to its high oxygen ion conductivity. ${ }^{5}$ Ceria has also received much attention in the area of three-way catalysts ${ }^{6}$ as oxygen promoters and in the area of catalysts for $\mathrm{H}_{2}$ production from fuels. ${ }^{7,8}$ Moreover, ceria has gained much attention for development of ultraviolet (UV) absorbent material, its absorption at about $400 \mathrm{~nm}$ being the strongest among all oxides. ${ }^{9}$

Several techniques including hydrothermal, ${ }^{10,11}$ reversemicellular, ${ }^{12}$ sonochemical, ${ }^{13}$ and those involving homogeneous precipitation ${ }^{14}$ have been used for the preparation of

*Authors to whom correspondence should be addressed. ceria particles. As a wet-chemical technique, the hydrothermal process has attracted a lot of attention since desired morphology can readily be produced at high purity by controlling parameters such as reaction time, reaction temperature, concentration, and type of reactant. Previously, Yang et al. ${ }^{15}$ reported the synthesis of cube-shaped $\mathrm{CeO}_{2}$ starting from cerium(III) nitrate and $\mathrm{NaOH}$ at different temperatures over $24 \mathrm{~h}$. The authors studied the effect of $[\mathrm{NaOH}]$ on the cubes and found that the size of the particles increased as the concentration was increased. The effect of $\mathrm{pH}$ on the crystallization of nanosized $\mathrm{CeO}_{2}$ was studied by $\mathrm{Li} \mathrm{W}-\mathrm{H}$ et al. ${ }^{16}$ It was claimed that the crystallites synthesized in an acidic hydrothermal medium are larger and more monodisperse than the ones produced in a neutral or alkaline medium. The effect of the counteranions of cerium source on $\mathrm{CeO}_{2}$ nanostructures was investigated by $\mathrm{Hu}$ et al. ${ }^{17}$ They reported that the synthesized nanorods converted into cubes by addition of $\mathrm{NO}_{3}^{-}$ions under hydrothermal conditions. When $\mathrm{NO}_{3}^{-}$ions were present in the solution, the dissolution-recrystallization process was largely improved. $\mathrm{NO}_{3}^{-}$ions act as both capping 
agent and an oxidizer during the formation of the $\mathrm{CeO}_{2}$ cubes. The influence of several other anions (such as $\mathrm{Br}^{-}$, $\mathrm{I}^{-}$, and $\mathrm{SO}_{4}^{2-}$ ) was also investigated showing that the basicity of the hydrothermal medium had a direct influence on the size and shape of the particles formed. However, there is lack of information on how of alkali bases affect the structure and morphology of $\mathrm{CeO}_{2}$ particles. Such bases, also known as mineralizers, are used as a source of alkalinity for the crystallization of metal oxides and increase the solubility of reagents to the levels sufficient for reactivity in a reasonable time ${ }^{18}$ Here, we investigate the influence of type and concentration of alkali base $(\mathrm{LiOH}, \mathrm{NaOH}, \mathrm{KOH})$ as well as reaction time and reaction temperature on the particle morphology and crystal growth of $\mathrm{CeO}_{2}$ particles. $\mathrm{X}$-ray diffractometry (XRD), scanning electron microscopy (SEM), high resolution transmission electron microscopy (HR-TEM), UV-vis spectroscopy, and photoluminescence (PL) spectroscopy were utilized for characterization. We have demonstrated that the size and shape of the particles can be controlled through a combination of time and temperature; and the growth kinetics can be modulated by the type of metal hydroxides.

\section{EXPERIMENTAL PROCEDURE}

\subsection{Experimental Method}

The cerium oxide nanocrystals were produced from the reaction of $0.5 \mathrm{~g}$ cerium nitrate hexahydrate $\left(\mathrm{Ce}\left(\mathrm{NO}_{3}\right)_{3}\right.$. $6 \mathrm{H}_{2} \mathrm{O}$ ) and a $10 \%$ solution of alkali metal hydroxide ( $\mathrm{MOH}: \mathrm{M}=\mathrm{Li}, \mathrm{Na}, \mathrm{K}$ ). The aqueous alkaline solutions were rapidly added to an aqueous solution of cerium nitrate inside a $23 \mathrm{~mL}$ teflon-lined stainless steel autoclave up to $50 \%$ of total available volume. The hydrothermal reactions were carried out at various temperatures from $120{ }^{\circ} \mathrm{C}$ to $240{ }^{\circ} \mathrm{C}$ with the reaction times being varied from $1 \mathrm{~h}$ to $24 \mathrm{~h}$. Each system was then allowed to cool to room temperature. The resulting particles were washed with $15 \mathrm{~mL}$ of water and separated by centrifugation at $6000 \mathrm{rpm}$ for $20 \mathrm{~min}$. The particles were obtained by drying the precipitates at $60{ }^{\circ} \mathrm{C}$ in air overnight.

\subsection{Characterization}

The crystal structure of the products was identified by X-ray powder diffraction (XRD recorded on a Philips-XperPro powder diffractometer with $\mathrm{Cu} \mathrm{K} \alpha$ radiation of $0.15406 \AA$ ). The morphology of the $\mathrm{CeO}_{2}$ nanocrystals was observed by scanning electron microscope (SEM-Philips XL-30S FEG, $5 \mathrm{kv}$ ) and transmission electron microscope (TEM-FEI Tecnai F-20 microscope). The optical properties were measured with a UV/VIS spectrometer (Varian Cary 50) equipped with a $1 \mathrm{~cm}$ quartz cuvette holder for liquid samples. Photoluminescence (PL) measurements were performed on a Varian Cary Eclipse Fluorescence
Spectrometer under excitation at $290 \mathrm{~nm}$. All absorbance and fluorometry measurements were done with the particles suspended in deionized water. Due to the low dispersibility of $\mathrm{CeO}_{2}$ nanoparticles in water, the samples were ultrasonically agitated for $10 \mathrm{~min}$ before measurement.

\section{RESULT AND DISCUSSION}

\subsection{Structural and Morphological Characterization of $\mathrm{CeO}_{2}$ particles}

Hydrothermal crystallization, in general, is based on a dissolution-precipitation mechanism of a precursor prepared from a metal salt using an alkaline solution as an oxygen source. ${ }^{19}$ In the particular case of $\mathrm{CeO}_{2}$, it was claimed that the precursor is amorphous, and that crystalline $\mathrm{CeO}_{2}$ is obtained when a hydrothermal process is applied where the precursor is homogenously dissolved and subsequently reprecipitated. ${ }^{15}$ However, in this study, we have demonstrated that the precursor is not amorphous and that the crystallization process begins during mixing of the reactant solutions at RT prior to hydrothermal treatment. Figure 1(a) presents the XRD pattern of a representative precursor prepared in the presence of $\mathrm{NaOH}$. The reflections are well indexed for $\mathrm{Ce}_{2} \mathrm{O}_{3}(\bullet), \mathrm{CeO}_{2}(\bullet)$, and as well as unreacted $\mathrm{Ce}\left(\mathrm{NO}_{3}\right)_{3}(\boldsymbol{\$})$. The presence of sharp reflections of both $\mathrm{Ce}_{2} \mathrm{O}_{3}$ and $\mathrm{CeO}_{2}$ clearly suggests that the precursor already contains the crystalline product

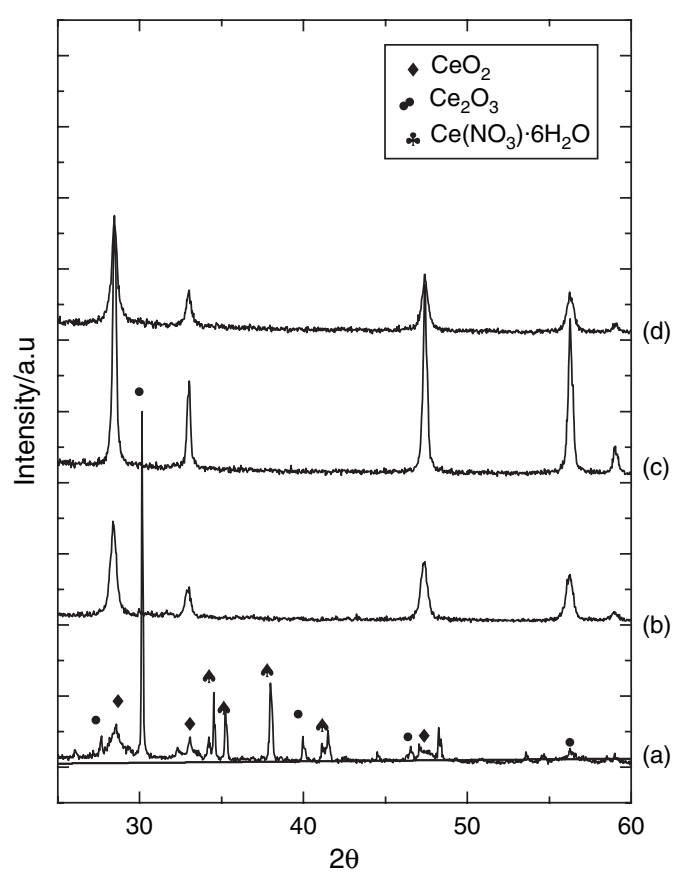

Fig. 1. (a) X-Ray Diffraction pattern of precursor precipitated prior to hydrothermal treatment. X-Ray Diffraction patterns of $\mathrm{CeO}_{2}$ powders synthesized at $240{ }^{\circ} \mathrm{C}$ for $24 \mathrm{~h}$ in the presence of (b) $\mathrm{KOH}$, (c) $\mathrm{NaOH}$, (d) $\mathrm{LiOH}$. 
(Supplementary Fig. S1). When hydrothermal treatment is applied at $240{ }^{\circ} \mathrm{C}$ for $24 \mathrm{~h}$ the signals of $\mathrm{Ce}\left(\mathrm{NO}_{3}\right)_{3}$ and $\mathrm{Ce}_{2} \mathrm{O}_{3}$ disappeared and the pure $\mathrm{CeO}_{2}$ phase was detected, regardless of the alkali base used. Particle morphology (size and shape) can be controlled by the type and concentration of $\mathrm{MOH}$, reaction time, and reaction temperature.

\subsubsection{Type of Alkali Base}

The effect of alkali metal hydroxides on the morphology of the precipitated $\mathrm{CeO}_{2}$ particles was examined. It was found that the formation and growth of $\mathrm{CeO}_{2}$ nanocubes were influenced significantly by the type of $\mathrm{MOH}$ used. The spectra (in Fig. 1) labeled as b, c, and d refer to the $\mathrm{CeO}_{2}$ particles prepared in the presence of $\mathrm{LiOH}, \mathrm{NaOH}$, and $\mathrm{KOH}$, respectively. All reflections in the spectra can be indexed to a face-centered cubic structure (JCPDS no 81-0792) with a lattice parameter of $5.412 \AA$. This value is slightly larger than the value of $5.410 \AA$ for the pure $\mathrm{CeO}_{2}$ phase as reported in the work of Tsunekawa et al. ${ }^{20}$ Figure 2 shows SEM images of $\mathrm{CeO}_{2}$ particles obtained by heating at $240{ }^{\circ} \mathrm{C}$ for $24 \mathrm{~h}$ separately with the alkali bases $\mathrm{LiOH}, \mathrm{NaOH}$, and $\mathrm{KOH}$. The particles all appear to have uniform cubic shape with different edge lengths depending on the counter-cation employed. The cubic shape is clearly observed for the relatively large particles produced in the presence of $\mathrm{NaOH}$. Figure 3 presents an overview of the high resolution TEM images as well as the selected area electron diffraction (SAED) patterns of the particles precipitated in the presence of $8 \mathrm{M} \mathrm{MOH}$. In contrast to the ones precipitated by $\mathrm{NaOH}$, the cubes prepared by $\mathrm{LiOH}$ and $\mathrm{KOH}$ have sharp corners. HRTEM images show clear view of lattice fringes with interplanar spacings of $0.22 \mathrm{~nm}$, revealing that the $\mathrm{CeO}_{2}$ nanocube is singlecrystalline. The crystalline nature of the resultant $\mathrm{CeO}_{2}$ cubes was also verified by their SAED pattern which was taken over an area of $21 \mathrm{~nm}$ in diameter. The pattern is basically a ring and can be indexed to fluorite structure, similarly XRD pattern.

Microscopy images, in principal, provide twodimensional views of the specimen. Therefore, the appearance of square faces in TEM images may originate from shapes other than cubes such as square plates or square prisms. To overcome this uncertainty, a representative particle was selected and the electron beam was tilted with respect to the plane upon which the specimen particle was located. The idea was to obtain a side-view image of the cube to be confident of the shape of the particles. Figure 4 shows TEM images of the representative $\mathrm{CeO}_{2}$ particles at different tilt angles in the range of $0^{\circ}$ to $56^{\circ}$. At $0^{\circ}$, the image presents a two-dimensional view. As the electron beam is tilted, the other edges of the structure begin to appear. A uniform cubic shape appears at $48^{\circ}$. This lends much support in showing that this representative particle has a uniform cubic shape.
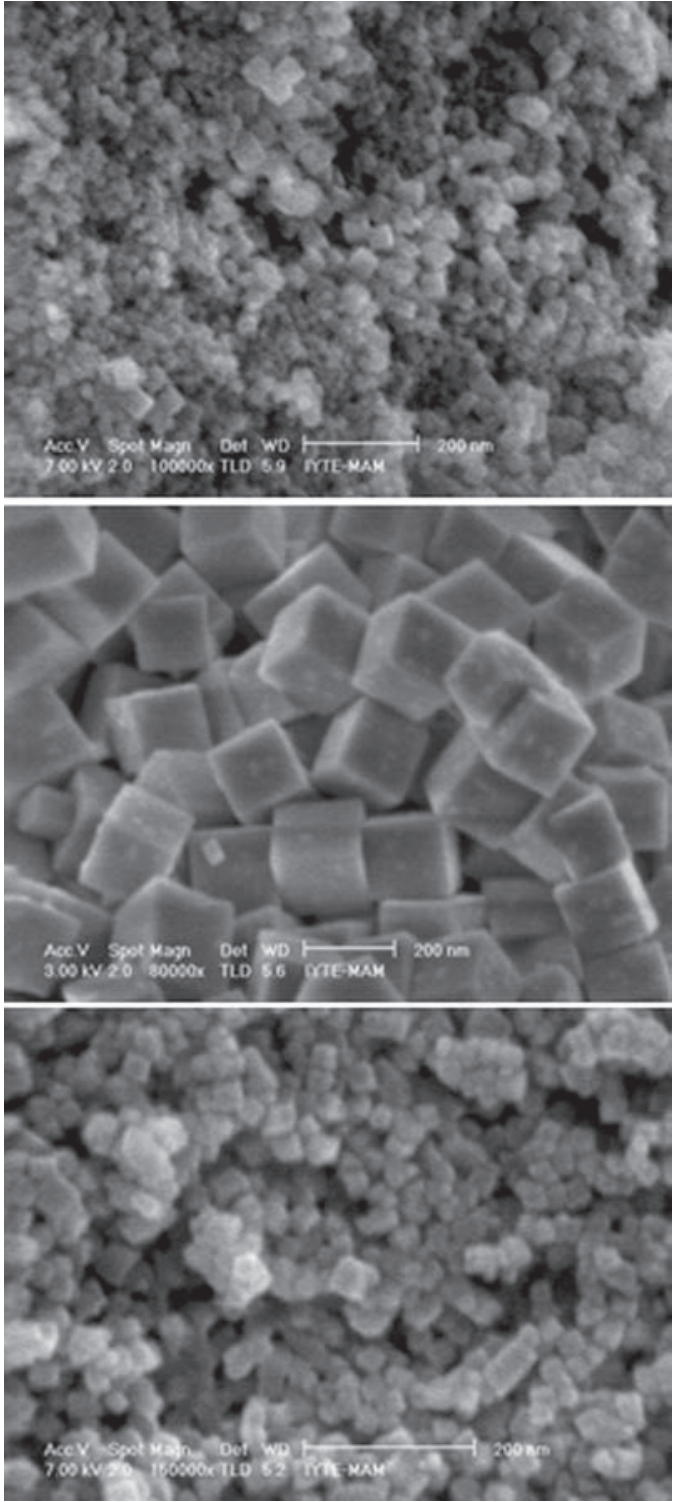

Fig. 2. SEM images of cerium oxide nanocubes produced at $240{ }^{\circ} \mathrm{C}$ for 24 h using $\mathrm{LiOH}, \mathrm{NaOH}$, and $\mathrm{KOH}$.

The edge length of the cubes was determined by statistical treatment of over 100 particles from both SEM and TEM images using Image $\mathrm{J}$ software. ${ }^{21}$ The distributions of edge length are shown in Figure 5. They exhibit Lorentzian distributions which is typical for crystal growth. The edge length distributions with respect to time are given in Figure S2. The edges of the particles were found to be in the nanoscale range when $\mathrm{LiOH}$ and $\mathrm{KOH}$ were used whereas they fall into the submicron range when $\mathrm{NaOH}$ was employed.

\subsubsection{Concentration}

The change in concentration was found to be one of the critical conditions for the formation of $\mathrm{CeO}_{2}$ particles. 

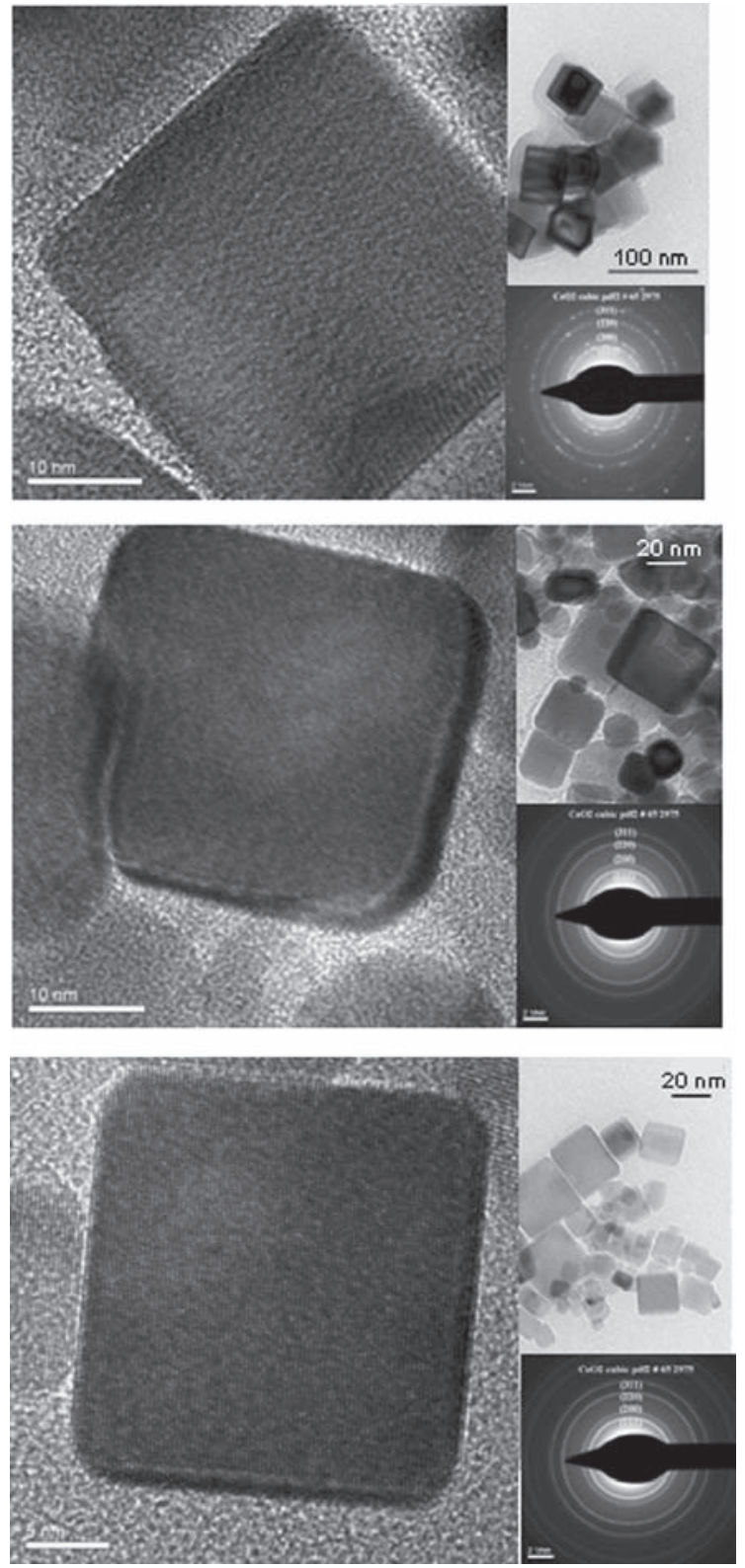

Fig. 3. TEM images of $\mathrm{CeO}_{2}$ nanocubes synthesized at $240{ }^{\circ} \mathrm{C}$ for $24 \mathrm{~h}$. The used base was (a) $\mathrm{LiOH}$, (b) $\mathrm{NaOH}$, (c) $\mathrm{KOH}$. Insets show HRTEM images and SAED patterns.

Variation of $\mathrm{MOH}$ concentration was performed while temperature and reaction time was fixed at $240{ }^{\circ} \mathrm{C}$ and $24 \mathrm{~h}$. Figure S3 (supporting information) shows SEM images of the particles precipitated in the presence of different concentrations of $\mathrm{MOH}$. As the concentration was increased from $0.1 \mathrm{M}$ to $8 \mathrm{M}, \mathrm{CeO}_{2}$ particles got larger and were uniform-shaped. The particles obtained at low concentrations were small and appeared to be more aggregated in the powder form. Independent of the chemistry of $\mathrm{MOH}$ used, larger cubes could be formed at higher temperature and higher concentration due to the Oswald ripening process. ${ }^{22}$

\subsubsection{Reaction Time}

Time-dependent experiments were carried out to monitor the evolving process by fixing the temperature of the reaction at $240{ }^{\circ} \mathrm{C}$ with $8 \mathrm{M} \mathrm{NaOH}$ alkali base for time intervals between 1 and $24 \mathrm{~h}$. The evolution of representative $\mathrm{CeO}_{2}$ particles precipitated by $\mathrm{NaOH}$ is shown in Figure 6. Initially, after $1 \mathrm{~h}$, the hydrothermal product became a mixture of rod-like and cubic particles. Extending the reaction time to $2 \mathrm{~h}$ favored the morphological transition whereby rod-like particles were converted to cubic ones. The same set of time-dependent experiments was performed for both $\mathrm{LiOH}$ and $\mathrm{KOH}$. In contrast to particles prepared by $\mathrm{NaOH}$, at $240{ }^{\circ} \mathrm{C}$, nanosized cubes are obtained in the presence of $\mathrm{LiOH}$ and $\mathrm{KOH}$ while rod-like particles were never realized even at $30 \mathrm{~min}$ of shorter reaction time.

\subsubsection{Reaction Temperature}

Figure 7 shows the change in morphology and size with respect to increase in temperature using $\mathrm{LiOH}$ as a representative case. Rod-shaped $\mathrm{CeO}_{2}$ nanoparticles were obtained at $120{ }^{\circ} \mathrm{C}$ for the all three alkali bases. As the temperature was increased, rod-shaped crystals gradually transformed into cubic-shaped crystals. Starting out as rod-shaped at $120{ }^{\circ} \mathrm{C}$, the crystals become cubic with heavy agglomeration at $160{ }^{\circ} \mathrm{C}$. Then at $200{ }^{\circ} \mathrm{C}$ the crystals are predominantly cube-shaped and finally at $240{ }^{\circ} \mathrm{C}$ well-shaped uniform cubes are formed. The results we present here are in agreement with the results reported by Yang et al. ${ }^{15}$ The authors demonstrated that control of the reaction temperature in hydrothermal synthesis of $\mathrm{CeO}_{2}$ particles is the most effective parameter that influences particle morphology. Based on the results given here, it is safe to say that the morphology of crystals is not only time-dependent but also temperature-dependent. Thus, a clear time-temperature superposition is confirmed.

\subsection{Particle Growth Kinetics}

The kinetics of particle growth was studied in order to fully understand the evolution of particles. For determination of growth kinetics, crystallite size as evaluated by observing line broadening of their XRD profiles. Average crystallite size was estimated from analysis of ceria by measuring the full-width-at-half-maximum (FWHM) of the most intense signal (111) using the Scherrer equation (Eq. (1)). ${ }^{23}$

$$
D=\frac{0.9 \lambda}{B \cos \theta_{\mathrm{B}}}
$$

where $\lambda$ is the wavelength of radiation, $B$ is the peak width defined as the full-width-at-half-maximum (FWHM) of the Bragg peak on the $2 \theta$ scale (in radians) and $\theta_{\mathrm{B}}$ is the Bragg angle. 

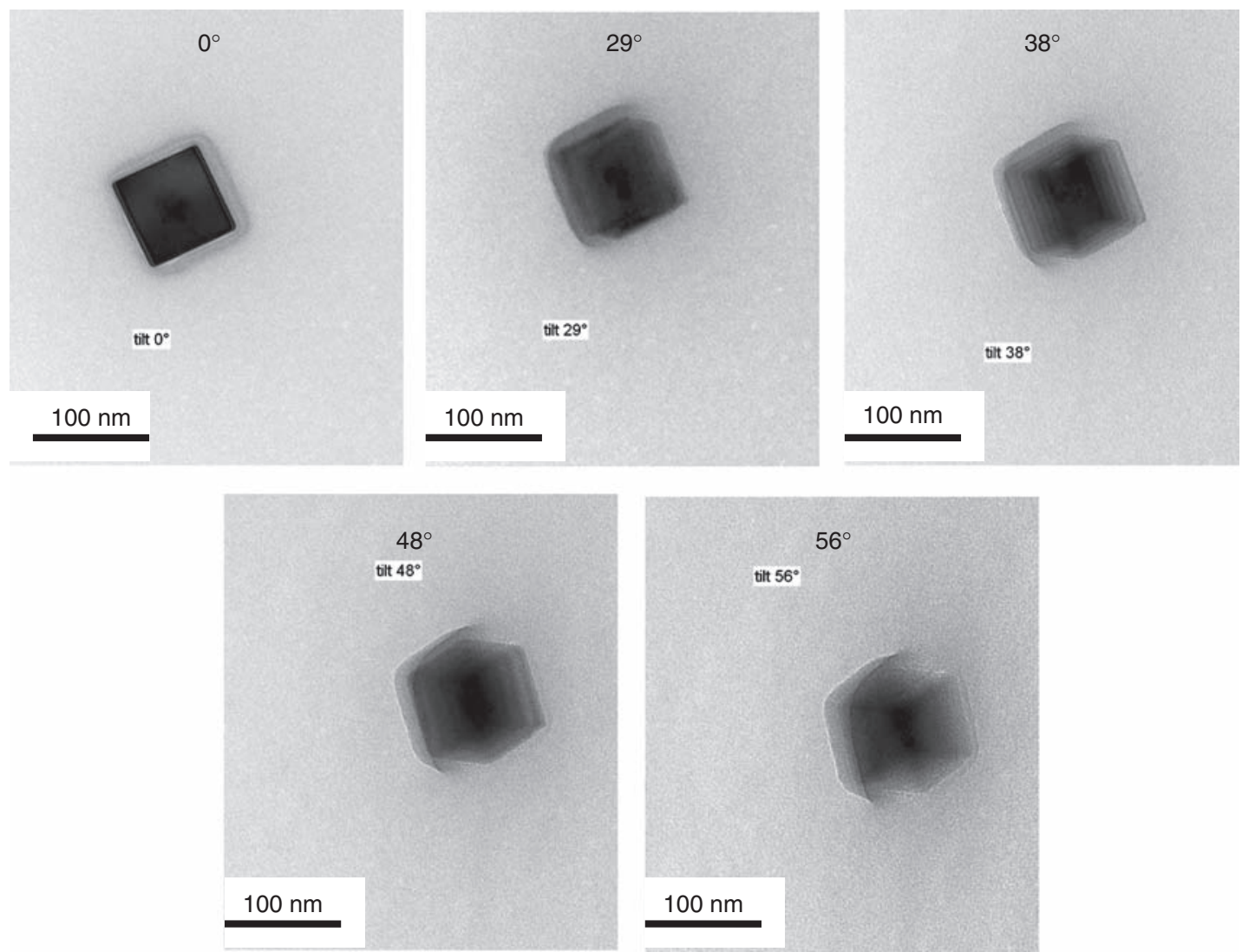

Fig. 4. Representative TEM images of $\mathrm{CeO}_{2}$ synthesized using $\mathrm{LiOH}$ from different angles proving three dimensional cubic structure of the $\mathrm{CeO}_{2}$ nanocrystals.

The progressive increase of the crystallite size of cubic ceria with concentration, time, and temperature for each mineralizer is shown in Figure 8. The concentration of alkali base in the reaction medium played an important role in the ultimate size of the resulting nanocrystals. When the base concentration is high enough, nanocrystals with high chemical potential can be synthesized, leading to larger $\mathrm{CeO}_{2}$ particles. Increasing the temperature of the

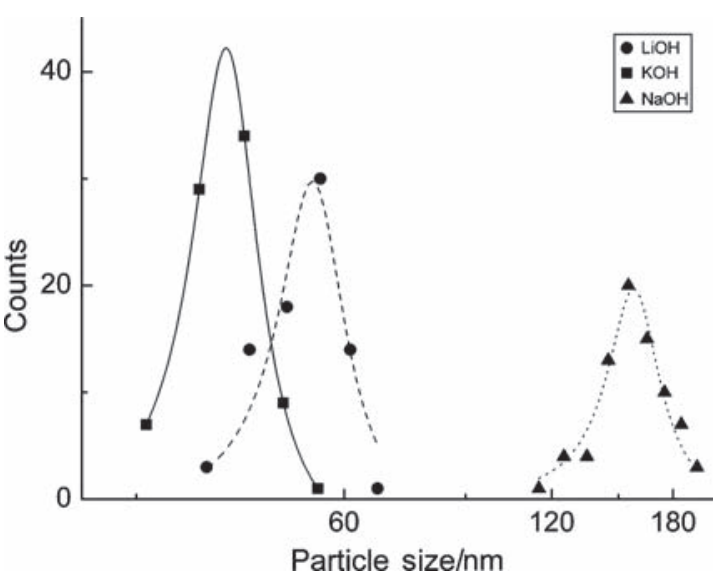

Fig. 5. Edge length distributions of $\mathrm{CeO}_{2}$ cubes prepared in the presence of $\mathrm{MOH}$ at $240{ }^{\circ} \mathrm{C}$ for $24 \mathrm{~h}$. hydrothermal synthesis results in an increase in the solubility of precursors and enhances the diffusion process of reactive species to/from the particle surface thereby resulting in larger crystals as well.

The grain-growth dependency on time was evaluated via the following equations:

$$
D^{n}-D_{o}^{n}=K t
$$

and

$$
K=K_{\mathrm{o}} \exp \left(\frac{-Q}{R T}\right)
$$

where $D^{n}$ is the grain size at time, $t ; D_{o}^{n}$ is the average grain size at time, $t=0 ; n$ is the grain exponent; $K$ is the rate constant; $K_{o}$ is the pre-exponential constant; $Q$ is the activation energy of grain growth; $R$ is the gas constant and $T$ is the absolute temperature. ${ }^{24}$ For the thermally activated processes $\left(D>D_{o}\right)$, these equations can be simplified as;

$$
D^{n}=K_{o} \exp \left(\frac{-Q}{R T}\right) t
$$

By plotting In $D$ versus $1 / T$ for a given time $t$, the slope will be equal to $-Q /(n R)$. The value of $n$ can be obtained by plotting In $D$ versus In $t$ for a given $T$. In a constant temperature range, $Q$ remains the same. Thus, the grain exponent $(n)$ can be found from the slope of the 

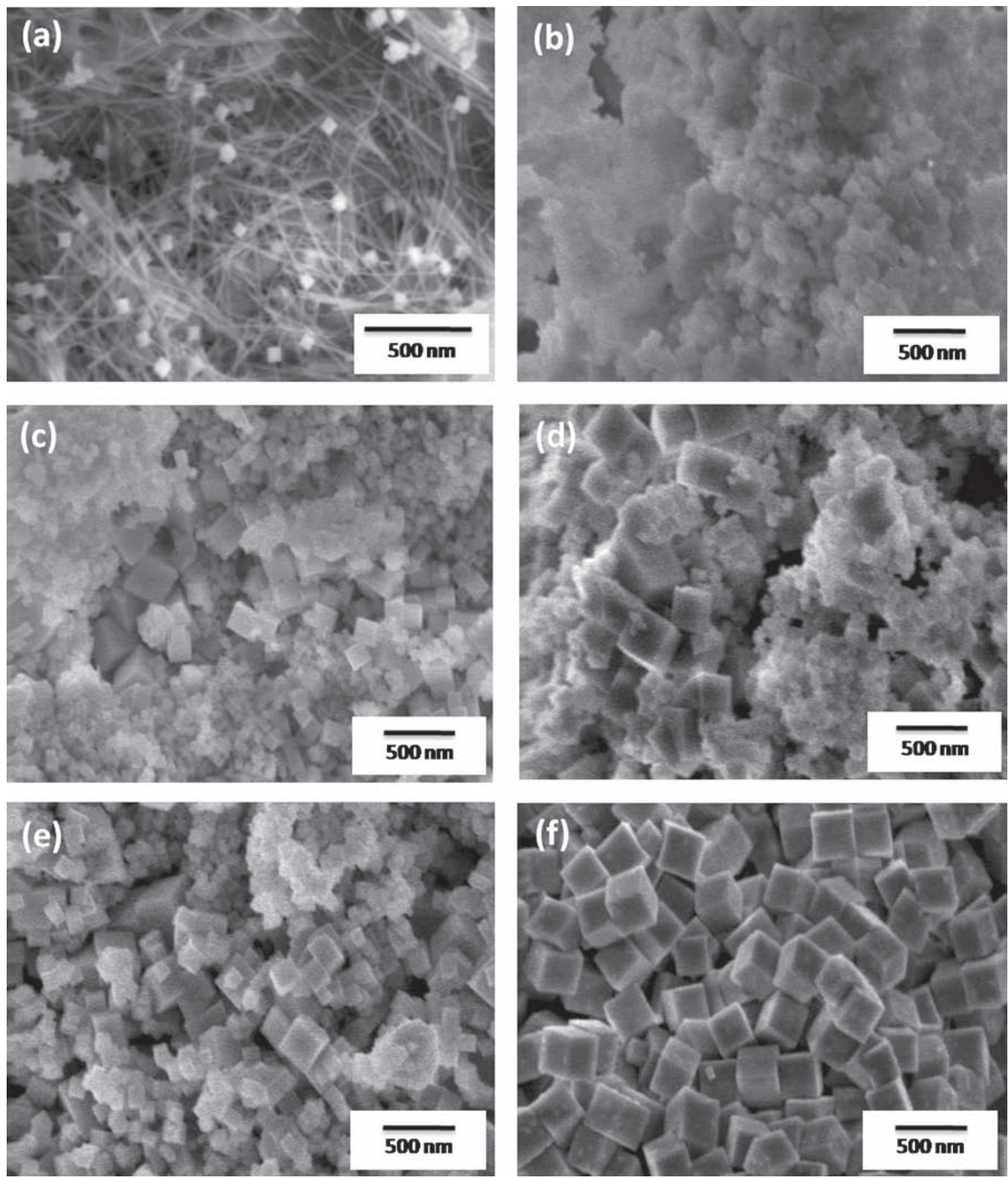

Fig. 6. Series SEM images of morphology evolution of cubic $\mathrm{CeO}_{2}$ nanoparticles with the stepwise prolonged reaction time (a) $1 \mathrm{~h}$, (b) $2 \mathrm{~h}$, (c) $4 \mathrm{~h}$, (d) $8 \mathrm{~h}$, (e) $16 \mathrm{~h}$, (f) $24 \mathrm{~h}$. The heating temperature was $240{ }^{\circ} \mathrm{C}$. The base used to obtain $\mathrm{CeO}_{2}$ was $\mathrm{NaOH}$.

plot of $\ln D$ versus $\ln t$ (Fig. 9). Based on these calculations, cerium oxide nanoparticles when synthesized using $\mathrm{LiOH}$ and $\mathrm{KOH}$ gave smaller particles with high $n$ values. However, nanoparticles synthesized with $\mathrm{NaOH}$ gave rather large particles with low values of $n$.

Dimensions (edge length and crystallite size), particle growth rate, activation energy, and product yield are presented in Table I. The yield of the hydrothermal reaction employed lies between 10-25\%, which varies with the type of alkali base used. Particle yield in chemical reactions, in general, is inversely proportional to the activation energy $(\mathrm{Q})$ required to convert reactant to products. According to collision theory, when $\mathrm{Q}$ is high, only a small fraction of reactant species is successfully converted to product. Similarly, a high yield is achieved at low Q. In the experiments presented here, the lowest yields were obtained for the particles produced in the presence of $\mathrm{KOH}$. Not surprisingly, $\mathrm{Q}$ is highest for these particles 

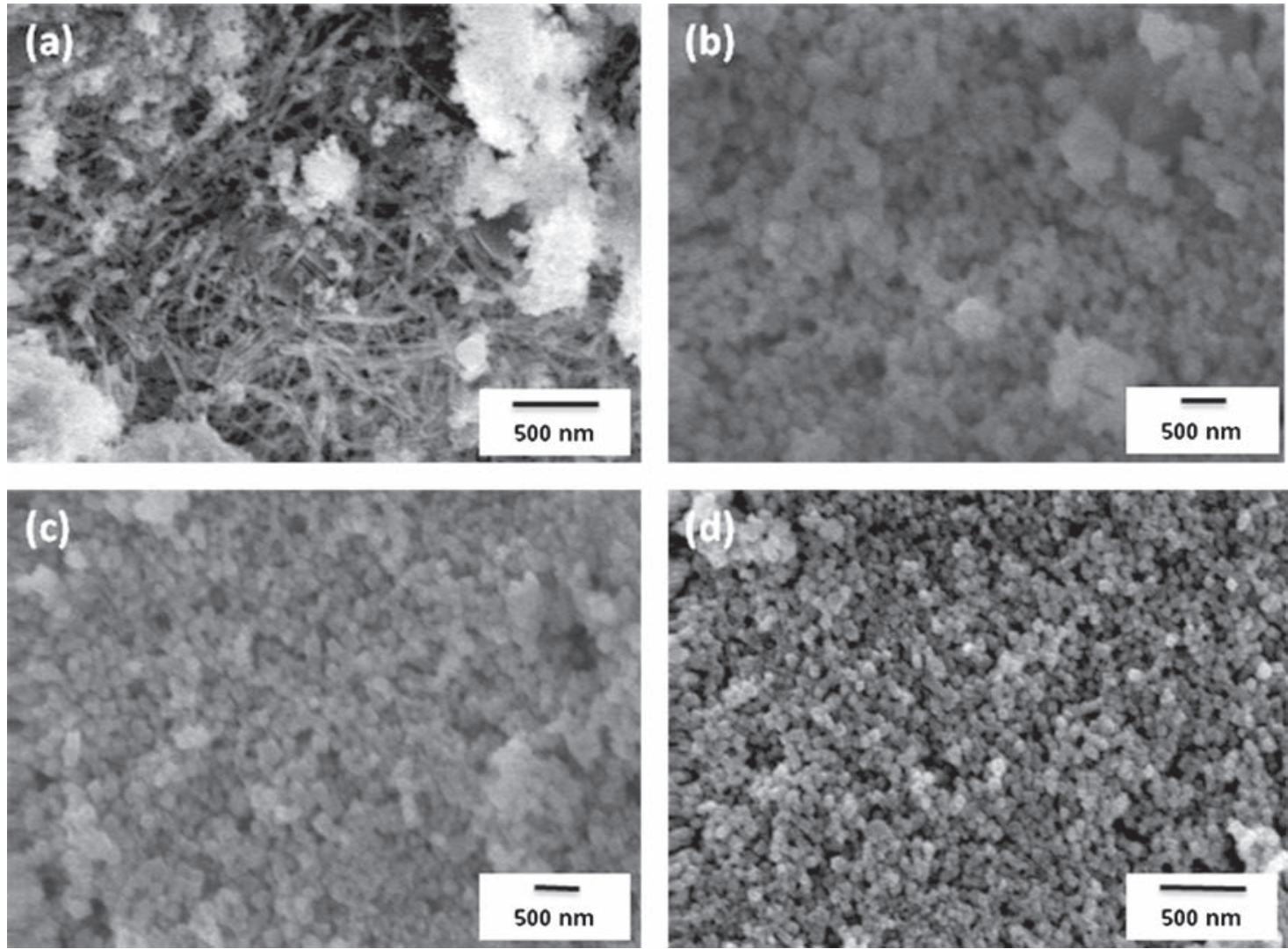

Fig. 7. SEM images of $\mathrm{CeO}_{2}$ synthesized using $\mathrm{LiOH}$ and the heating temperature was (a) $120{ }^{\circ} \mathrm{C}$, (b) $160{ }^{\circ} \mathrm{C}$, (c) $200{ }^{\circ} \mathrm{C}$ and (d) $240{ }^{\circ} \mathrm{C}$ for $24 \mathrm{~h}$.

as estimated from Eq. (4). However, using $\mathrm{LiOH}$ permits the highest yield due to its comparably lower (in fact lowest) Q.

All the crystallites do not grow to the same size because the internal crystallites are restricted by the presence of neighboring crystallites. They grow to fill all vacant voids. On the other hand, surface crystallites have less restraint thus leaving them free to grow away from the particle surface and reach the maximum size achievable for a given set of reaction conditions. The growth of different planes can be observed by comparing the intensity of corresponding reflections in the X-ray diffractograms. Here, the growth of crystallites is not symmetrical, because growth rate of the two prominent planes, $\{200\}$ and $\{111\}$ are not equal. ${ }^{27,28}$ Measurement of FWHM peak width allows the calculation of the separation distance between crystallite faces. This results in the ability to model the actual shape of the crystallite. The intensity ratio of reflections from the (200) and (111) planes were changed with reaction time causing the shape of ceria to change from polyhedron to cube. The growth of the (111) plane occurs because its free energy is lower than that of the (200) plane during crystal growth. The crystal changes its shape and chemistry from rod-like to hexagon (truncated octahedron) and finally to truncated cubic $\mathrm{CeO}_{2}$ submicron particles. The change in curvature of the corners of the cubes (Fig. 3) can be followed by the change in the intensity ratio of the diffraction signals of the (200) and (100) planes. Figure 10 shows the relationship of crystallite size with the intensity ratio of $(200) /(111)$ at different total reaction times. The intensity ratio of $(200) /(111)$ decreases when going from $\mathrm{LiOH}$ to $\mathrm{KOH}$ and $\mathrm{NaOH}$. The growth rate of the (200) plane is faster than that of the (100) plane in this reaction process. The ratio of the $(200) /(111)$ plane is highest for $\mathrm{LiOH}$, resulting in nanocubes with sharper corners. In the case of $\mathrm{NaOH}$ (possessing the smallest (200)/(111) ratio), the $\mathrm{CeO}_{2}$ nanocubes exposed predominantly the $\{200\}$ cubic facets with truncation of the corners exposing the $\{111\}$ facets.

\subsection{Discussion on Formation of $\mathrm{CeO}_{2}$ Particles}

Submicron-sized $\mathrm{CeO}_{2}$ crystals with well-defined shapes (rod-like or cubic) have been carefully prepared through a regular hydrothermal reaction taking place between $\left(\mathrm{CeNO}_{3}\right)_{3}$ and $\mathrm{MOH}$. Generally speaking, the growth of crystals is a non-equilibrium phenomenon. Different external conditions may change the course of the crystal growth and effecting particle morphology. Previously, Peng suggested that the concentration of existing monomer in the growth solution plays a key role in the determination and evolution of the shapes of the resulting crystals. ${ }^{27}$ At high monomer concentrations, crystals grow in preferentially one direction, yielding rod-shaped particles. The elongated 

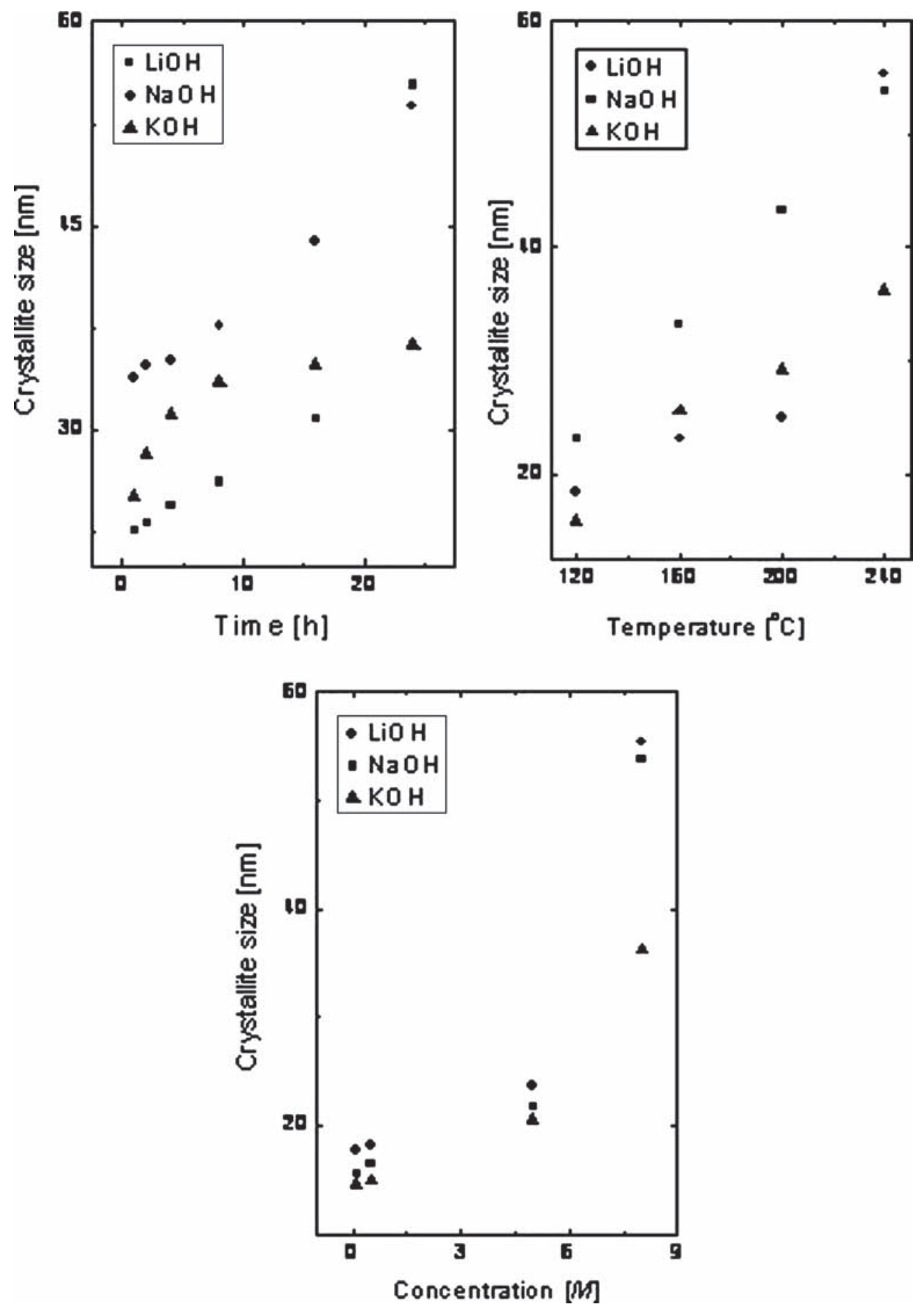

Fig. 8. Crystallite sizes based on (a) concentration, (b) reaction time, and (c) reaction temperature.

shape can be transformed into more spherical shapes if the monomer concentration in solution is lowered. The results we have obtained were consistent with this suggested model. At the beginning of the reaction, the concentration of monomer is the highest during the course of particle precipitation. Anisotropic $\mathrm{Ce}(\mathrm{OH})_{3}$ nuclei are formed once the $\mathrm{Ce}^{3+}$ ions are mixed with the $\mathrm{MOH}$ solution. $\mathrm{NO}_{3}^{-}$ions can possibly oxidize cerium (III) ions in the system to $\mathrm{CeO}_{2}$ species. ${ }^{17}$ Rod-like particles with an aspect ratio of about 10 were obtained at $120{ }^{\circ} \mathrm{C}$. The concentration of monomer is depleted by nucleation and growth of the rods. At higher reaction temperature (to 160$240{ }^{\circ} \mathrm{C}$ ), the dissolution/recrystallization process is initiated. The strong character of an alkali medium triggers this equilibrium process. The end points of the rods impart significant instability to dissolution, thus the dissolution might 


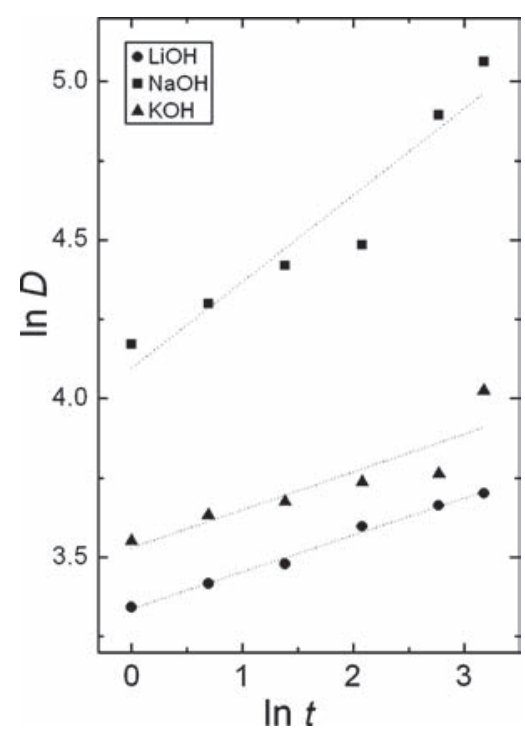

Fig. 9. Plot of In $D$ as a function of In $t$ for $\mathrm{CeO}_{2}$ particles.

occur at both end points. ${ }^{17}$ Eventually, $\mathrm{CeO}_{2}$ rods were converted into well-defined $\mathrm{CeO}_{2}$ polyhedron particles with $\{200\}$ and $\{111\}$ facets. The growth rate of $\{200\}$ appeared to be higher than that of $\{111\}$. Enlargement of $\{200\}$ with respect to $\{111\}$ led to cube-shaped $\mathrm{CeO}_{2}$ particles with $\{200\}$ facets. The entire morphological development based on the results obtained from microscopy and detailed diffraction was summarized graphically as in Scheme 1.

According to classical theories of crystallization, the shape-controlled growth of crystals is determined by the relative specific surface energy of each face or facets of the crystal. ${ }^{28}$ Generally, the face with the higher density of surface atoms is blocked by a surfactant adsorbed during the crystal growth of colloidal crystals, and the growth along this facet is therefore considerably restricted. ${ }^{28}$ No additional surfactant was used in our case; rather, the reaction mixture contains only reactants required for a simple hydrothermal method. Consequently, this reaction offers a really convenient and suitable path for mass production of well-defined $\mathrm{CeO}_{2}$ particles in terms of size and shape.

Table I. Dimensions of particles obtained at $240^{\circ} \mathrm{C}$ within $24 \mathrm{~h}$ of reaction time in the presence of $8 \mathrm{M} \mathrm{MOH}$, particle growth rate, activation energy, and yield of hydrothermal synthesis.

\begin{tabular}{lccccc}
\hline $\begin{array}{l}\text { Type of } \\
\text { alkali } \\
\text { base }\end{array}$ & $\begin{array}{c}\text { Mean of } \\
\text { edge length } \\
\text { distribution } \\
(\mathrm{nm})^{a}\end{array}$ & $\begin{array}{c}\text { Average } \\
\text { crystallite } \\
\text { size } \\
(\mathrm{nm})^{b}\end{array}$ & $\begin{array}{c}\text { Particle } \\
\text { growth rate } \\
(n \text { value })^{c}\end{array}$ & $\mathrm{Q}_{(\mathrm{kJ} / \mathrm{mol})^{d}}$ & Yield, \% $^{e}$ \\
\hline $\mathrm{LiOH}$ & 41 & 22 & 8.6 & 43.0 & 24.7 \\
$\mathrm{NaOH}$ & 158 & 54 & 3.7 & 113.8 & 10.2 \\
$\mathrm{KOH}$ & 56 & 36 & 13.1 & 150.9 & 9.3 \\
\hline
\end{tabular}

${ }^{a}$ From TEM images.

${ }^{b}$ Line broadening of XRD signals as calculated via Eq. (1).

${ }^{c}$ Derived from Eq. (2).

${ }^{d}$ Derived from Eq. (4).

${ }^{e}$ Gravimetry.

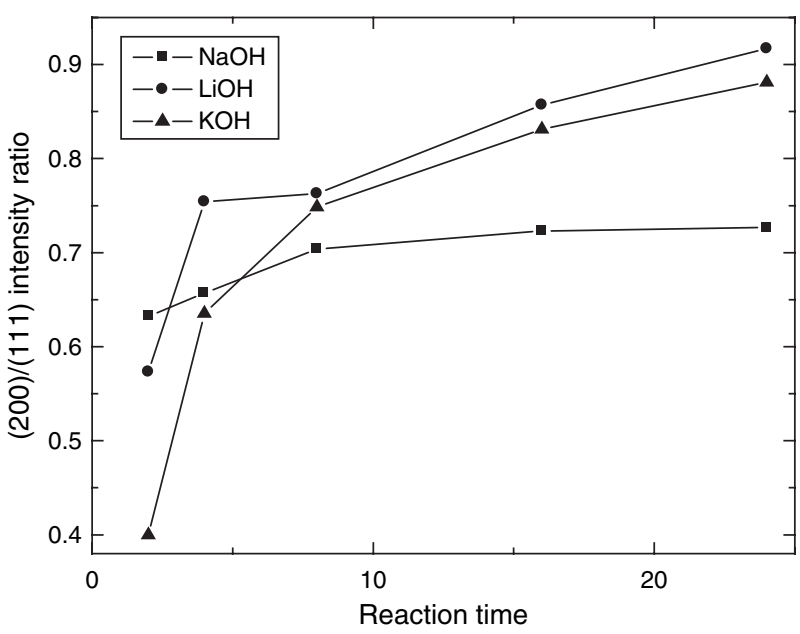

Fig. 10. Intensity ratio of $(200) /(100)$ of the powders prepared in the presence of $\mathrm{MOH}$.

Kinetics of particle growth was systematically studied for each MOH. Different growth kinetics as well as growth mechanisms were found. There are two possible ways of describing the growth of $\mathrm{CeO}_{2}$ crystals. These are aggregation and Ostwald ripening. In the former, a certain number of growing particles randomly adhere together to form large particle aggregates with nonuniform shape. Microscope images of particles clearly show uniform particle shape; thus the mechanism of aggregation is ruled out. The latter, Ostwald ripening, can be defined as a thermodynamically driven spontaneous process in which small particles dissolve, reduce in size and disappear. Atoms detach from small particles and diffuse to attach to large particles causing large particles to grow at the expense of small particles. From examination of the formed particles in the product, information can be obtained by only with regard to morphology, but not about the development of the particles. Therefore, it is hard to elucidate a growth mechanism of particles from only the final shape and structure. However, a comparision of physical dimensions obtained from microscopy and line broadening in XRD patterns may give a hint about the growth mechanism. The crystallite dimension was comparable to the size of the particles observed in microscopy for the ones prepared using $\mathrm{LiOH}$ and $\mathrm{KOH}$. The values of $n$ for $\mathrm{KOH}$ and $\mathrm{LiOH}$ are 13.1 and 8.4, respectively, and associated with growth by surface diffusion. This mechanism involves dissolution of small particles. The solubility product of the initial precipitate is, $K_{\text {sp }}\left(\mathrm{Ce}(\mathrm{OH})_{4}\right)=2 \times 10^{-48}$, quite small compared to frequently observed precipitated products, for example $K_{\text {sp }}(\mathrm{AgCl})=1.8 \times 10^{-10} .{ }^{16}$ So, the dissolution of particles, and therefore, the rate of growth will be low and the particles remain smaller. In contrast, for the particles obtained in the presence of $\mathrm{NaOH}$, the size of the particles observed by microscopy showed them to be nearly three times larger than the results from line broadening indicate. The particles grew by lattice or grain-boundary 


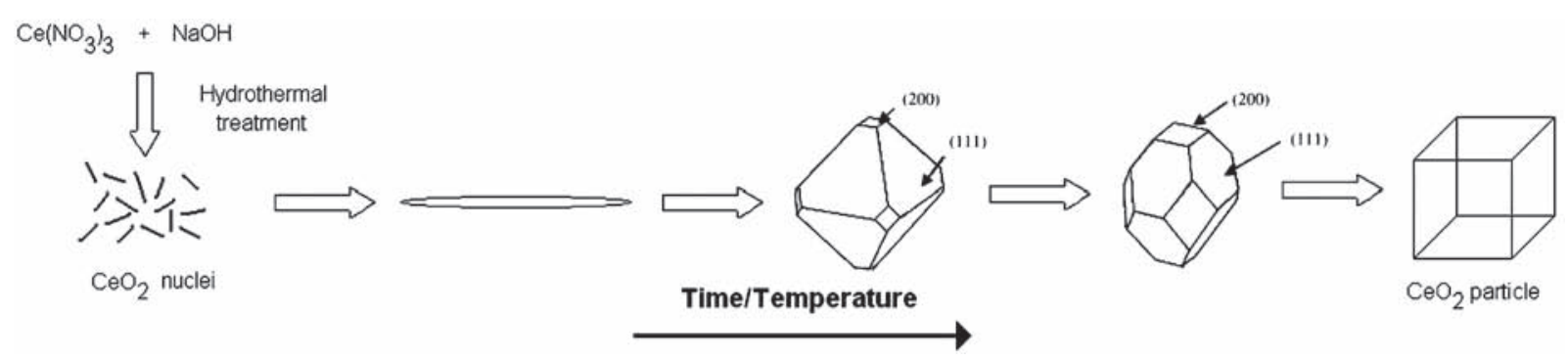

Scheme 1. Graphical abstract to expain the development particle morphology during hydrothermal synthesis of $\mathrm{CeO}_{2}$ cubes.
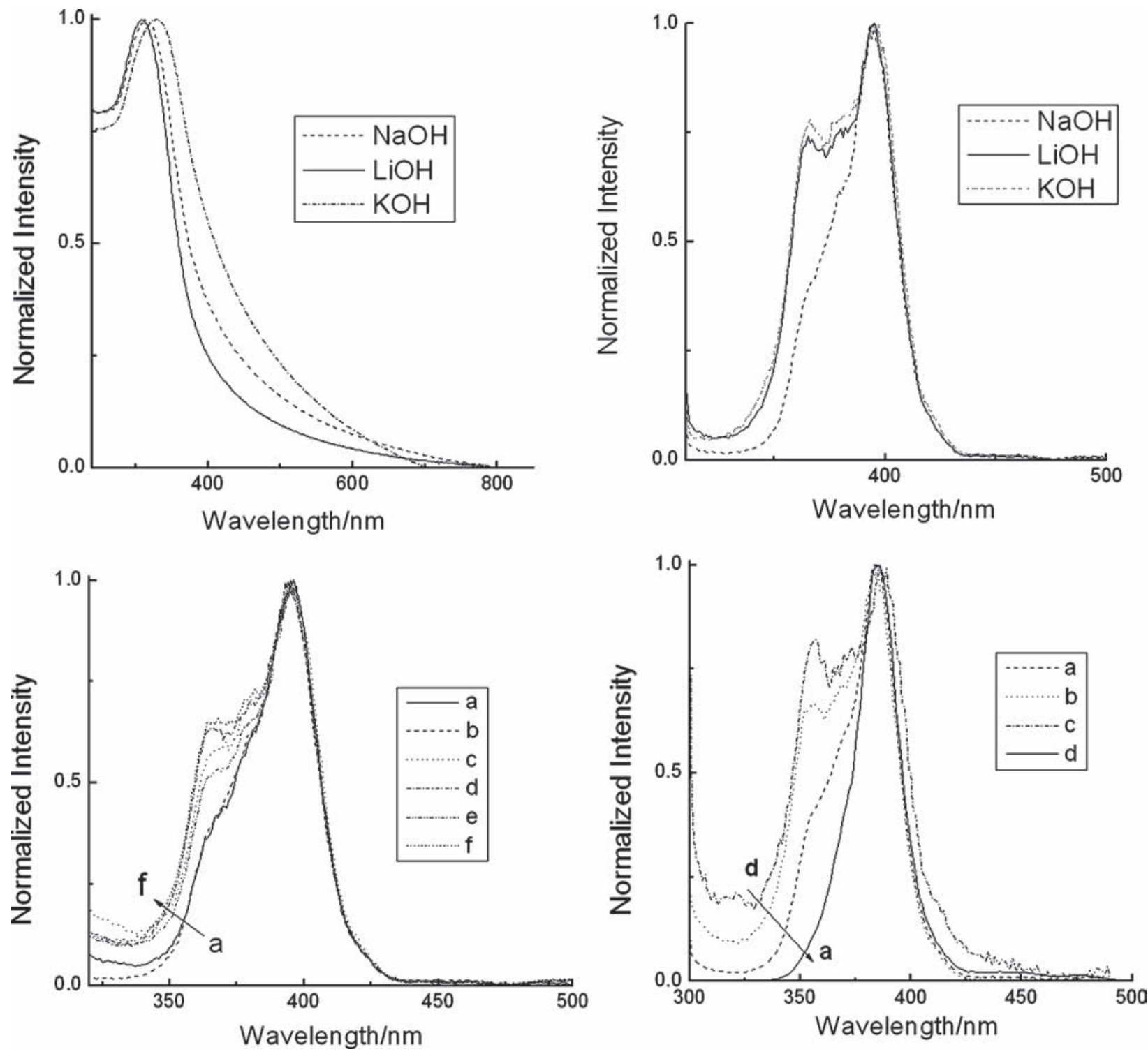

Fig. 11. (a) The UV-VIS absorbance spectrum of $\mathrm{CeO}_{2}$ nanoparticles obtained at $240{ }^{\circ} \mathrm{C}$ for $24 \mathrm{~h}$. The concentration of dispersions $1.05 \times 10^{-3} \mathrm{M}$. Inset is the band-gap values for bases. (b) Room temperature fluorescence spectra of the $\mathrm{CeO}_{2}$ nanoparticles dispersions with different alkali bases of $\mathrm{LiOH}, \mathrm{NaOH}$ and $\mathrm{KOH}$. The concentration of dispersions $1.05 \times 10^{-3} \mathrm{M}$ and the excited wavelength $290 \mathrm{~nm}$. (c) Room temperature fluorescence spectra of the $\mathrm{CeO}_{2}$ nanoparticles dispersions with different reaction times (a) $24 \mathrm{~h}$, (b) $16 \mathrm{~h}$, (c) $8 \mathrm{~h}$, (d) $4 \mathrm{~h}$, (e) $2 \mathrm{~h}$, (f) $1 \mathrm{~h}$. Excitation wavelength $290 \mathrm{~nm}$. (d) Room temperature fluorescence spectra of the $\mathrm{CeO}_{2}$ nanoparticles dispersions with different reaction temperatures (a) $240{ }^{\circ} \mathrm{C}$, (b) $200{ }^{\circ} \mathrm{C}$, (c) $160{ }^{\circ} \mathrm{C}$, (d) $120^{\circ} \mathrm{C}$. Excitation wavelength $290 \mathrm{~nm}$. 
diffusion of three (3) individual growing nuclei and were found to be larger compared to the ones prepared by $\mathrm{LiOH}$ and $\mathrm{NaOH}$ under identical preparation conditions. The $n$ value of growth rate is 3.7. Studies on grain-growth kinetics in the literature point out similar results. Chen and Chen found pure $\mathrm{CeO}_{2}$ grain-growth, obtained by homogeneous precipitation, as $n=2 .{ }^{29}$ Zhang et al. found the grain-growth of nanoparticles, as prepared by the conventional mixed-oxide method to have $n=3 .^{30}$

\subsection{Optical Properties of $\mathrm{CeO}_{2}$ Nanoparticles}

Figure 11 shows the absorption spectra of $\mathrm{CeO}_{2}$ nanoparticles synthesized in the presence of $\mathrm{MOH}$ at $240{ }^{\circ} \mathrm{C}$ for $24 \mathrm{~h}$. The concentration of the dispersive solutions was $1.05 \mathrm{mM}$. A well-defined clear absorption peak located at $315 \mathrm{~nm}$ was observed for the particles precipitated from each of these bases. The bandgap energies were estimated from the cut-off wavelength as measured at the point where the tangent crossed the wavelength axis. Measured absorption peaks were $307 \mathrm{~nm}, 314 \mathrm{~nm}$, and $327 \mathrm{~nm}$, corresponding to bandgaps of $3.38 \mathrm{eV}, 3.17 \mathrm{eV}$, and $2.98 \mathrm{eV}$ for $\mathrm{LiOH}, \mathrm{NaOH}$, and $\mathrm{KOH}$, respectively. These bandgap values are consistent with the literature. ${ }^{31,32}$

The chemical nature of mineralizers influences the structural arrangement of atoms during crystallization. Considerable research has been focused on the fluorescence properties of nanomaterials because fluorescence may reveal the presence of crystalline defects resulting from the synthesis process. In this context, photoluminescence emission spectra of pure $\mathrm{CeO}_{2}$ particles precipitated in the presence of $\mathrm{MOH}$ at various temperatures, reaction times, and concentrations were recorded for the suspension of the particles in $\mathrm{H}_{2} \mathrm{O}$. The particle suspensions were stable for the time required for the measurements. Additionally, independent measurements of solid-state spectra showed comparable results and confirmed the validity of our experimental method. The emission spectra of the $\mathrm{CeO}_{2}$ nanoparticles as measured at an excitation wavelength of $290 \mathrm{~nm}(4.28 \mathrm{eV})$, show two emission signals at $400 \mathrm{~nm}(3.10 \mathrm{eV})$ and $370 \mathrm{~nm}(3.27 \mathrm{eV})$. The first signal can be attributed to the band-edge exciton annihilation, whereas the origin of the second signal is open to debate. One of the common approaches for the possible explanation of this signal is ascribed to the presence of $\mathrm{Ce}^{3+}$ in the crystal structure of the matrix of $\mathrm{Ce}^{4+} .33$

Figure 11 presents normalized emission of the particles precipitated from the hydrothermal precursor at different reaction times (Fig. 11(c)) and different temperatures (Fig. 11(d)). For all samples, the intensity of emissions at $400 \mathrm{~nm}$ and $370 \mathrm{~nm}$ is enhanced as the temperature increases. However, the relative intensity of the second emission with respect to the emission which resulted from excitation annihilation is remarkably reduced. The $\mathrm{Ce}$ (III) defect states are considered to be hexagonal crystal phase. Increasing temperature favors the hexagonal crystal structure over the face-centered cubic structure although the latter is thermodynamically more stable. Therefore, the tendency towards the face-centered cubic structure at higher temperature subsequently leads to a decrease in the number of defect sites at higher temperatures. A similar result is obtained from the particles obtained at different reaction times when the temperature was held constant. As the reaction time is extended, the intensity of this signal at $370 \mathrm{~nm}$ diminishes gradually. By increasing reaction temperature and extending reaction time the nucleation process is improved, leading to greater completeness of crystal growth and thus allowing the particles to find a more favorable lattice (i.e. the quality of the resultant crystals is improved). Therefore, this result suggests that emission at $370 \mathrm{~nm}$ can be a result of existence of $\mathrm{Ce}(\mathrm{III})$.

\section{CONCLUSION}

We have reported hydrothermal synthesis of monodisperse $\mathrm{CeO}_{2}$ particles. At $120{ }^{\circ} \mathrm{C}$, the particles have a rod-like morphology. Increasing the temperature of the reaction medium to between 160 and $240{ }^{\circ} \mathrm{C}$ forces the particles to take on a cubic shape via a dissolution-recrystallization process. Edge length is highly tailorable with remarkable precision through choice of alkali base and by adjusting reaction time, reaction temperature, and concentration of MOH. In general, all alkali metals employed here show similar behavior in terms of their influence on morphological development and optical features. However, they differ in their affect on particle growth kinetics; the mechanism underlying this growth seems to be cation-dependent. The particles grow through surface diffusion when using $\mathrm{KOH}$ and $\mathrm{LiOH}$ whereas they follow growth via a grainboundary or lattice-diffusion process when formed in the presence of $\mathrm{NaOH}$. A more detailed study including all alkali- and as well as alkaline-earth bases that control particle morphology and growth kinetics is underway.

\section{SUPPLEMENTARY FIGURES}

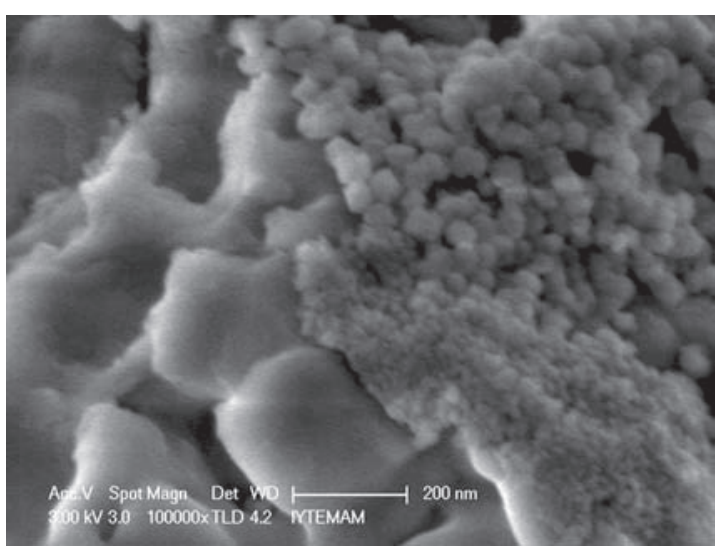

Fig. S1. SEM images of cerium oxide nanocubes produced prior to hydrothermal treatment at room temperature. 

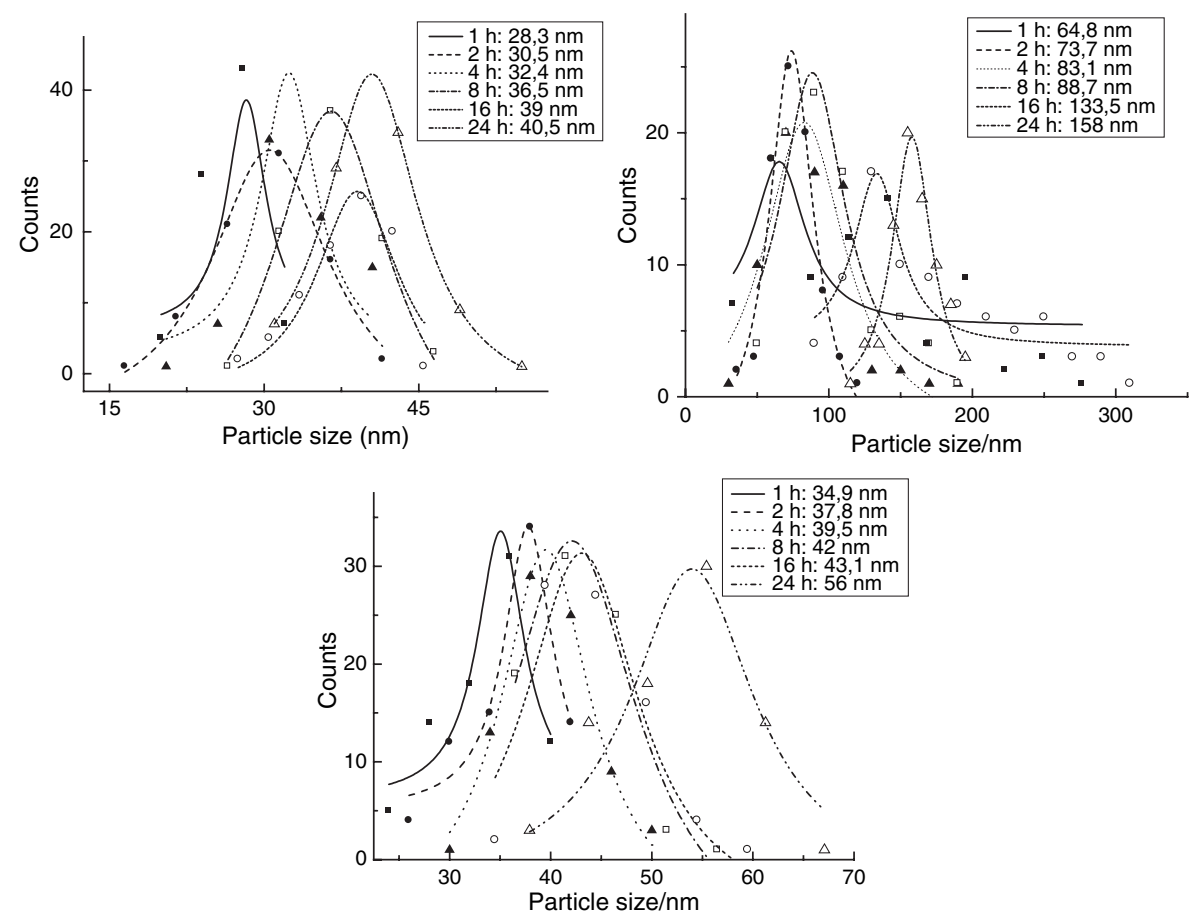

Fig. S2. Particle size distribution of $\mathrm{CeO}_{2}$ nanoparticles in presence of (a) $\mathrm{LiOH}$, (b) $\mathrm{NaOH}$, (c) $\mathrm{KOH}$.
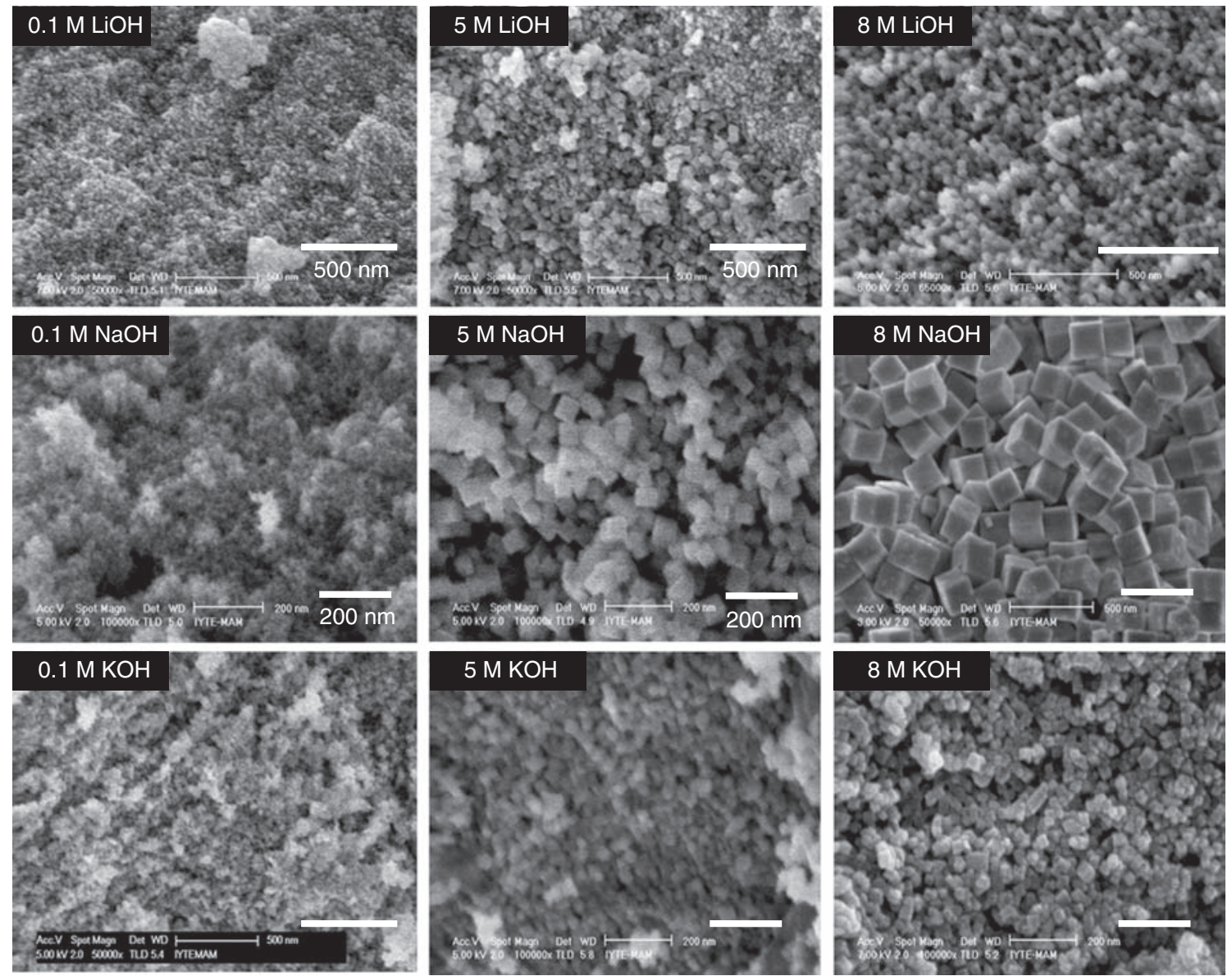

Fig. S3. SEM images of cerium oxide nanocubes produced at $240{ }^{\circ} \mathrm{C}$ for $24 \mathrm{~h}$. The concentrations and sort of the bases are given in the images. 


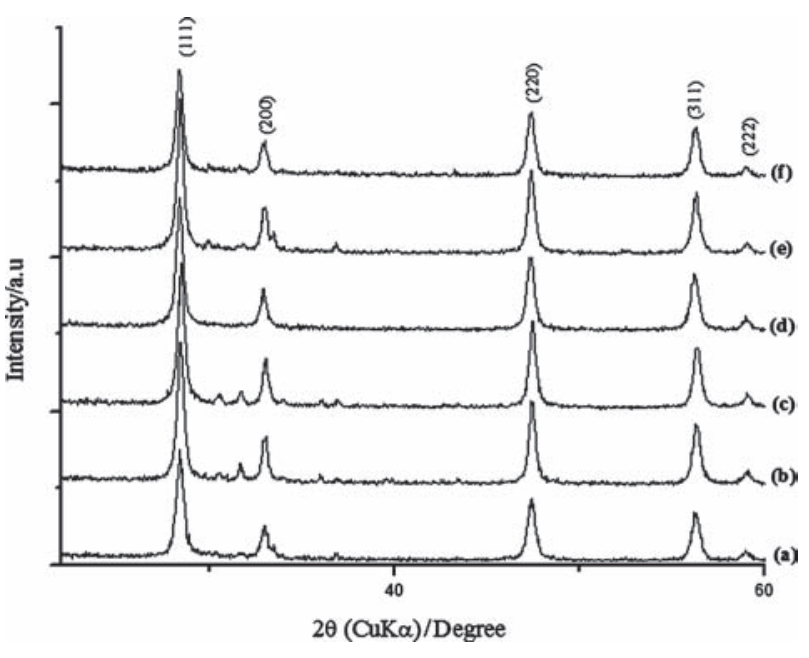

Fig. S4. XRD patterns of $\mathrm{CeO}_{2}$ nanoparticles with the stepwise prolonged reaction time (a) $1 \mathrm{~h}$, (b) $2 \mathrm{~h}$, (c) $4 \mathrm{~h}$, (d) $8 \mathrm{~h}$, (e) $16 \mathrm{~h}$, (f) $24 \mathrm{~h}$. The heating temperature was $240{ }^{\circ} \mathrm{C}$. The base used to obtain $\mathrm{CeO}_{2}$ was $\mathrm{LiOH}$.

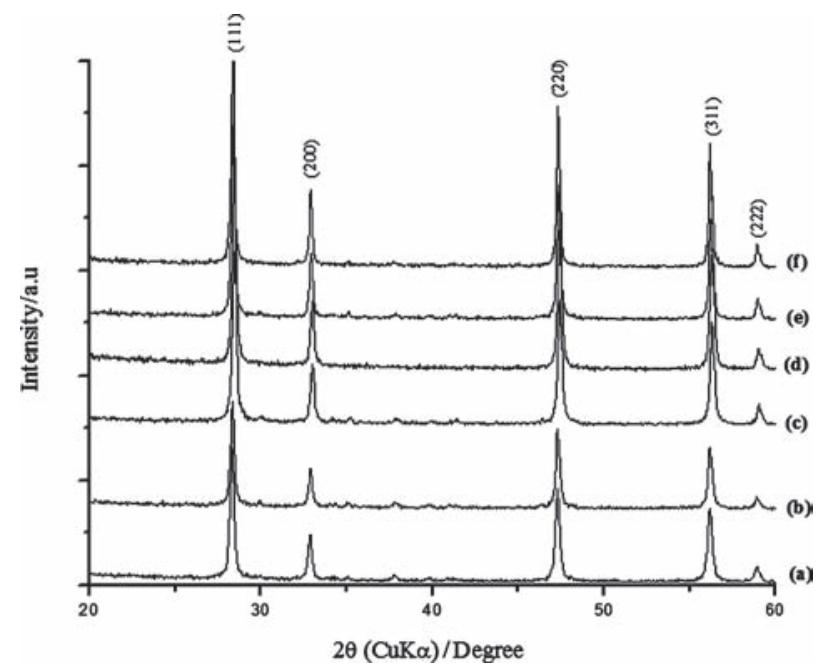

Fig. S5. XRD patterns of $\mathrm{CeO}_{2}$ nanoparticles with the stepwise prolonged reaction time (a) $1 \mathrm{~h}$, (b) $2 \mathrm{~h}$, (c) $4 \mathrm{~h}$, (d) $8 \mathrm{~h}$, (e) $16 \mathrm{~h}$, (f) $24 \mathrm{~h}$. The heating temperature was $240{ }^{\circ} \mathrm{C}$. The base used to obtain $\mathrm{CeO}_{2}$ was $\mathrm{NaOH}$.

Acknowledgments: This work was supported by the Scientific and Technological Research Council of Turkey (grant no: TBAG 108T664) and Izmir Institute of Technology (2009IYTE26). The authors thank Dr. I. Lieberwirth of MPIP for TEM images and Dr. R. C. Eanes for his scientific and proofreading suggestions during the preparation of the written manuscript.

\section{References and Notes}

1. T.-W. Jun, J.-S. Choi, and J. Cheon, Angew. Chem. Int. Ed. 45, 2 (2006).

2. M. B. Fernando and G. P. W Marques, J. Am. Ceram. Soc. 74, 598 (2005).

3. B. Vodungbo, Y. Zheng, F. Vidal, D. Demaille, and V. H. Etgens, Appl. Phys. Lett. 90, 62510 (2007).

4. T. K. K. Alston, M. Palin, M. Prica, and P. Windibank, J. Power Sources 71, 271 (1998).

5. T. U. K. Hibino and Y. Kuwahara, Solid State Ionics 93, 309 (1997).

6. M. V. M. Boaro, C. de Leitenburg, G. Dolcetti, and A. Trovarelli, Catal. Today 77, 407 (2003).

7. A. Trovarelli, Catalysis Reviews-Science and Engineering 38, 439 (1996).

8. A. Trovarelli, C. Leitenburg, M. Boaro, and G. Dolcetti, Catal. Today 353 (1999).

9. N. Imanaka, M. Masui, H. Hirai, and G. Adachi, Chem. Mater. 15, 2289 (2003).

10. K. A. T. Byrappa, Progr. Cryst. Growth Char. Mater. 53, 117 (2007).

11. H. Mai, L. Sun, Y. Zhang, R. Si, W. Feng, H. Zhang, H. Liu, and C. Yan, J. Phys. Chem. B 109, 24380 (2005).

12. S. Sathyamurthy, K. J. Leonard, R. T. Dabestani, and M. P. Paranthaman, Nanotechnology 16, 1960 (2005).

13. H. Wang, J. J. Zhu, J. M. Zhu, X. H. Liao, S. Xu, T. Ding, and H. Y. Chen, Phys. Chem. Chem. Phys. 4, 3794 (2002).

14. X. D. Zhou, W. Huebner, and H. U. Anderson, Appl. Phys. Lett. 80, 3814 (2002).

15. Z. Yang, K. Zhou, X. Liu, Q. Tian, D. Lu, and S. Yang, Nanotechnology 18, 185606 (2007).

16. N.-C. Wu, E.-W. Shi, Y.-Q. Zheng, and W.-J. Li, J. Am. Ceram. Soc. 85, 2462 (2002).

17. Q. Z. F. Wu, P. Xiao, H. Tao, X. Wang, and Z. Hu, J. Phys. Chem. C 112, 17076 (2008).

18. G. Philip and W. L. Jessop, Chemical Synthesis Using Supercritical Fluids, Wiley-Vch Verlag GmbH (1999).

19. J.-S. Lee and S.-C. Choi, Mater. Lett. 58, 390 (2004).

20. S. Tsunekawa, K. Ishikawa, Z. Q. Li, Y. Kawazoe, and A. Kasuya, Phys. Rev. Lett. 85, 3440 (2000).

21. Public domain software to be downloaded from http://rsb.info.gov/ij (National Institute of Health).

22. R. D. Vengrenovich, Y. V. Gudyma, and S. V. Yarema, Semiconductors 35, 1378 (2001).

23. H. P. Klug and L. E. Alexander, X-ray Diffraction Procedures: For Crystaline and Amorphous Materials, Wiley \& Sons, New York (1974).

24. E. R. Leite, M. A. L. Nobre, M. Cerqueira, E. Longo, and J. A. Varela J. Am. Ceram. Soc. 80, 2649 (1997).

25. N. B. Kirk and J. V. Wood, J. Mater. Sci. 30, 2171 (1995).

26. M.-S. Tsai and X.-Z. Xiao, J. Cryst. Growth 289, 351 (2006).

27. X. Peng, Adv. Mater. 15, 459 (2003).

28. Z. L. Wang and X. Feng, J. Phys. Chem. B 107, 13563 (2003).

29. P. L. Chen and I. W. Chen, J. Am. Ceram. Soc. 79, 1793 (1996).

30. T. Zhang, P. Hing, H. Huang, and J. Kilner, Mater. Lett. 57, 507 (2002).

31. T. Masui, K. Fujiwara, K. Machida, and G. Adachi, Chem. Mater. 9, 2197 (1997).

32. L. Yin, Y. Wang, G. Pang, Y. Koltypin, and A. Gedanken, J. Colloid Interface Sci. 246, 78 (2002).

33. Z. F. Ma, G. C. Liang, and J. S. Liang, J. Rare Earths 22, 247 (2004).

Received: 14 June 2010. Accepted: 15 June 2010. 